\title{
Some variations of EM algorithms for Marshall-Olkin bivariate Pareto distribution with location and scale
}

\author{
Arabin Kumar Dey · Biplab Paul
}

the date of receipt and acceptance should be inserted later

\begin{abstract}
Recently Asimit et. al (Asimit et al. (2016)) used an EM algorithm to estimate Marshall-Olkin bivariate Pareto distribution. The distribution has seven parameters. We describe few alternative approaches of EM algorithm. A numerical simulation is performed to verify the performance of different proposed algorithms. A real-life data analysis is also shown for illustrative purposes.
\end{abstract}

Keywords Joint probability density function, Bivariate Pareto distribution, EM algorithm, Pseudo likelihood function

\section{Introduction}

Bivariate Pareto has several forms. In this paper we study Marshal-Olkin formulation (Marshall and Olkin (1967)) of this distribution which includes both location and scale parameters. In a very recent paper, Asimit et al. (Asimit et al. (2010)) used EM algorithm to estimate the parameters of this distribution. We adapt few more variations of the same. We observe that some of our proposed algorithms either works equally well or outperforms their algorithm. We also present some real life data analysis which is absent in their paper.

\footnotetext{
A. K. Dey

Department of Mathematics,

IIT Guwahati,

Guwahati, India

Assam

Tel.: +91361-258-4620

E-mail: arabin@iitg.ac.in

B. Paul

Department of Mathematics,

IIT Guwahati,

Guwahati, India

E-mail: biplab.paul@iitg.ac.in
} 
In this paper our focus is on singular bivariate Pareto distribution whose both the marginals have Pareto type-II distribution. We can obtain bivariate Pareto distribution considering peak over threshold method in a bivariate data. The distribution has wide application in modeling data related to finance, insurance, environmental sciences and internet network. Any analysis based on this distribution requires efficient techniques for estimating parameters of the distribution. We consider a more generalized set up including location and scale parameters in formation of Marshal-Olkin bivariate Pareto distribution. This dependence structure can be described by well-known Marshall-Olkin copula too [Nelsen (2007), Marshall and Olkin (1967), Yeh (2000), Yeh (2004)]. We propose few innovative ways to implement EM algorithm.

A random variable $\mathrm{X}$ is said to have Pareto of second kind, i.e. $X \sim P a(I I)(\mu, \sigma, \alpha)$ if it has the survival function

$$
\bar{F}_{X}(x ; \mu, \sigma, \alpha)=P(X>x)=\left(1+\frac{x-\mu}{\sigma}\right)^{-\alpha}
$$

and the probability density function (pdf)

$$
f(x ; \mu, \sigma, \alpha)=\frac{\alpha}{\sigma}\left(1+\frac{x-\mu}{\sigma}\right)^{-\alpha-1}
$$

with $x>\mu \in \mathscr{R}, \sigma>0$ and $\alpha>0$.

Kundu and Gupta (2009), Kundu and Gupta (2010), Kundu and Dey (2009) used EM algorithm for estimating parameters of different bivariate distributions, e.g. bivariate generalized exponential, bivariate Weibull etc. Dey and Kundu (2012) performed discrimination between bivariate Weibull and bivariate generalized exponential distributions where they used parameter estimation through EM algorithm. Sarhan and Balakrishnan (2007) considered estimation issues in Sarhan and Balakrishnan bivariate distribution with extra scale parameter in their model. Many works have been done on multivariate Pareto distribution too [Hanagal (1996), Yeh (2000), Yeh (2004), Asimit et al. (2010)]. Statistical Inference of multivariate Pareto distribution through EM algorithm is attempted by Asimit et al. (2016). This paper also deals with seven parameters including location and scale as its parameters. We handle the same problem in a slightly different manner.

We arrange the paper in the following way. In section 2 we keep the MarshallOlkin bivariate Pareto formulation and some of its properties. In section 3, we describe our proposed EM algorithms. Some simulation results show the performance of the algorithm in section 4. In section 5 we show the data analysis. Finally we conclude the paper in section 6 .

\section{Formulation of Marshal-Olkin bivariate Pareto}

Let $U_{0}, U_{1}$ and $U_{2}$ be three independent univariate type-II Pareto distributions $\mathrm{Pa}(I I)\left(0,1, \alpha_{0}\right)$, $\mathrm{Pa}(I I)\left(\mu_{1}, \sigma_{1}, \alpha_{1}\right)$ and $\mathrm{Pa}(I I)\left(\mu_{2}, \sigma_{2}, \alpha_{2}\right)$.

We define $X_{1}=\min \left\{\sigma_{1} U_{0}+\mu_{1}, U_{1}\right\}$ and $X_{2}=\min \left\{\sigma_{2} U_{0}+\mu_{2}, U_{2}\right\}$. We can show that $\left(X_{1}, X_{2}\right)$ jointly follow bivariate Pareto distribution of second kind, we call it as $B V P A\left(\mu_{1}, \mu_{2}, \sigma_{1}, \sigma_{2}, \alpha_{0}, \alpha_{1}, \alpha_{2}\right)$ 
The joint distribution can be given by

$$
f\left(x_{1}, x_{2}\right)= \begin{cases}f_{1}\left(x_{1}, x_{2}\right) & \text { if } \frac{x_{1}-\mu_{1}}{\sigma_{1}}<\frac{x_{2}-\mu_{2}}{\sigma_{2}} \\ f_{2}\left(x_{1}, x_{2}\right) & \text { if } \frac{x_{1}-\mu_{1}}{\sigma_{1}}>\frac{x_{2}-\mu_{2}}{\sigma_{2}} \\ f_{0}(x) & \text { if } \frac{x_{1}-\mu_{1}}{\sigma_{1}}=\frac{x_{2}-\mu_{2}}{\sigma_{2}}=x\end{cases}
$$

where

$$
\begin{aligned}
f_{1}\left(x_{1}, x_{2}\right) & =\frac{\alpha_{1}}{\sigma_{1} \sigma_{2}}\left(\alpha_{0}+\alpha_{2}\right)\left(1+\frac{x_{2}-\mu_{2}}{\sigma_{2}}\right)^{-\left(\alpha_{0}+\alpha_{2}+1\right)}\left(1+\frac{x_{1}-\mu_{1}}{\sigma_{1}}\right)^{-\left(\alpha_{1}+1\right)} \\
f_{2}\left(x_{1}, x_{2}\right) & =\frac{\alpha_{2}}{\sigma_{1} \sigma_{2}}\left(\alpha_{0}+\alpha_{1}\right)\left(1+\frac{x_{1}-\mu_{1}}{\sigma_{1}}\right)^{-\left(\alpha_{0}+\alpha_{1}+1\right)}\left(1+\frac{x_{2}-\mu_{2}}{\sigma_{2}}\right)^{-\left(\alpha_{2}+1\right)} \\
f_{0}(x) & =\alpha_{0}(1+x)^{-\left(\alpha_{1}+\alpha_{2}+\alpha_{0}+1\right)}
\end{aligned}
$$

It can be shown that

1. The distribution of $X_{j}$ is $\operatorname{Pa}(I I)\left(\mu_{j}, \sigma_{j}, \alpha_{0 j}\right), \alpha_{0 j}=\alpha_{0}+\alpha_{j}$.

2. Distribution of the minimum is also Pareto distribution when $U_{0}, U_{1}$ and $U_{2}$ has same location and scale parameter.

3. Maximum Likelihood estimate of $\mu, \sigma, \alpha$, the parameters of univariate Pareto can be given based on the data set $Y_{1}, Y_{2}, \cdots, Y_{n}$ as $: \mu=\min \left\{Y_{1}, Y_{2}, \cdots, Y_{n}\right\}$, whereas estimates of $\alpha$ and $\sigma$ can be obtained by solving the fixed point iterations :

$$
\sigma=\frac{\alpha+1}{n} \sum_{i=1}^{n} \frac{\left(x_{i}-\mu\right)}{\left(1+\frac{x_{i}-\mu}{\sigma}\right)}
$$

where,

$$
\alpha=\frac{n}{\sum_{i=1}^{n} \ln \left(1+\frac{x_{i}-\mu}{\sigma}\right)}
$$

Surface and contour plots of the absolutely continuous part of the pdf are shown in Figure-1 and Figure-2 respectively. The following four different sets of parameters provide four subfigures in each figure. $\xi_{1}: \mu_{1}=0, \mu_{2}=0, \sigma_{1}=1, \sigma_{2}=0.5, \alpha_{0}=$ $1, \alpha_{1}=0.3, \alpha_{2}=1.4 ; \xi_{2}: \mu_{1}=1, \mu_{2}=2, \sigma_{1}=0.4, \sigma_{2}=0.5, \alpha_{0}=2, \alpha_{1}=1.2, \alpha_{2}=$ $1.4 ; \xi_{3}: \mu_{1}=0, \mu_{2}=0, \sigma_{1}=1.4, \sigma_{2}=0.5, \alpha_{0}=1, \alpha_{1}=1, \alpha_{2}=1.4 ; \xi_{4}: \mu_{1}=0, \mu_{2}=$ $0, \sigma_{1}=1.4, \sigma_{2}=0.5, \alpha_{0}=2, \alpha_{1}=0.4, \alpha_{2}=0.5$. 


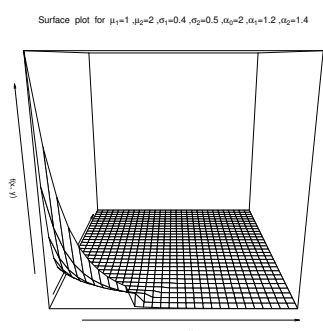

(a) $\xi_{1}$

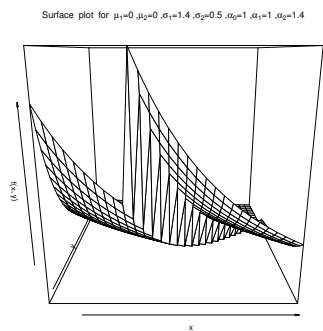

(c) $\xi_{3}$

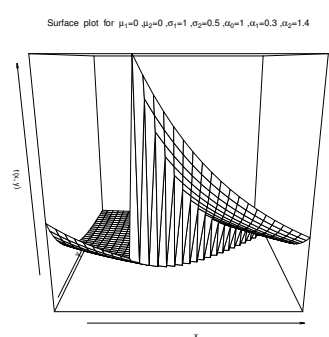

(b) $\xi_{2}$

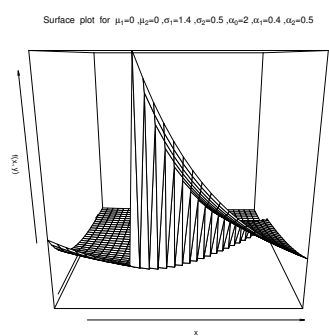

(d) $\xi_{4}$

Fig. 1 Surface plots for pdf of BVPA 


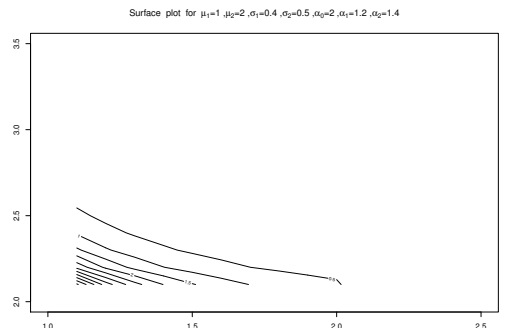

(a) $\xi_{1}$

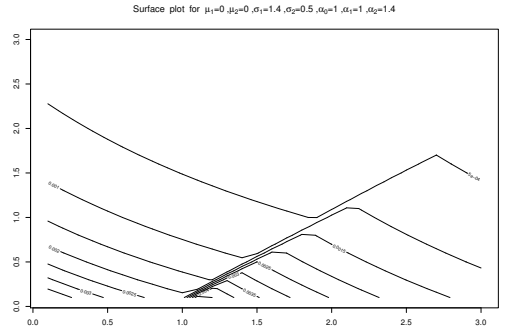

(c) $\xi_{3}$

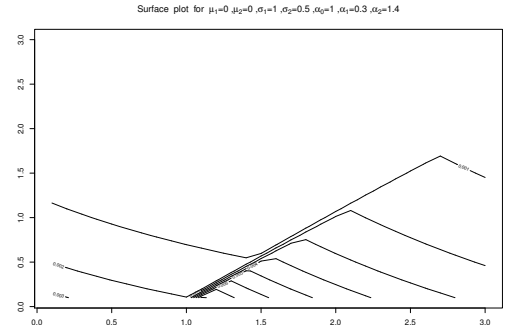

(b) $\xi_{2}$

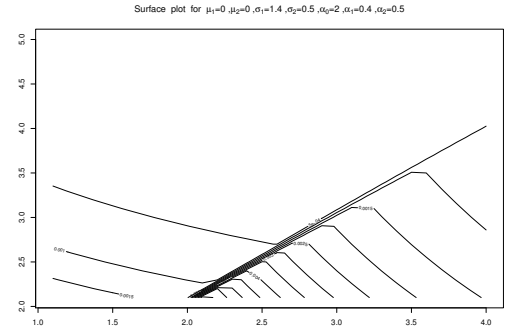

(d) $\xi_{4}$

Fig. 2 Contour plots for pdf of BVPA

\section{EM-algorithm}

Let us assume $\mu_{1}, \mu_{2}, \sigma_{1}$ and $\sigma_{2}$ are known. Now we divide our data into three parts

$I_{0}=\left\{\left(x_{1 i}, x_{2 i}\right): \frac{x_{1 i}-\mu_{1}}{\sigma_{1}}=\frac{x_{2 i}-\mu_{2}}{\sigma_{2}}\right\}, I_{1}=\left\{\left(x_{1 i}, x_{2 i}\right): \frac{x_{1 i}-\mu_{1}}{\sigma_{1}}<\frac{x_{2 i}-\mu_{2}}{\sigma_{2}}\right\}, I_{2}=\left\{\left(x_{1 i}, x_{2 i}\right):\right.$ $\left.\frac{x_{1 i}-\mu_{1}}{\sigma_{1}}>\frac{x_{2 i}-\mu_{2}}{\sigma_{2}}\right\}$

Usual Likelihood function for the parameters of the BVPA can be written as

$$
\begin{aligned}
& L\left(\mu_{1}, \mu_{2}, \sigma_{1}, \sigma_{2}, \alpha_{0}, \alpha_{1}, \alpha_{2}\right) \\
= & n_{1} \ln \alpha_{1}+n_{1} \ln \left(\alpha_{0}+\alpha_{2}\right)-n_{1} \ln \sigma_{1}-n_{1} \ln \sigma_{2} \\
- & \left(\alpha_{0}+\alpha_{2}+1\right) \sum_{i \in I_{1}}^{n} \ln \left(1+\frac{x_{2 i}-\mu_{2}}{\sigma_{2}}\right)-\left(\alpha_{1}+1\right) \sum_{i \in I_{1}} \ln \left(1+\frac{x_{1 i}-\mu_{1}}{\sigma_{1}}\right) \\
- & n_{2} \ln \sigma_{1}-n_{2} \ln \sigma_{2}+n_{2} \ln \alpha_{2}+n_{2} \ln \left(\alpha_{0}+\alpha_{1}\right) \\
- & \left(\alpha_{0}+\alpha_{1}+1\right) \sum_{i \in I_{2}}^{n} \ln \left(1+\frac{x_{1 i}-\mu_{1}}{\sigma_{1}}\right)-\left(\alpha_{2}+1\right) \sum_{i \in I_{2}}^{n} \ln \left(1+\frac{x_{2 i}-\mu_{2}}{\sigma_{2}}\right) \\
+ & n_{0} \ln \alpha_{0}-n_{0} \ln \sigma_{1}-\left(\alpha_{0}+\alpha_{1}+\alpha_{2}+1\right) \sum_{i \in I_{0}}^{n} \ln \left(1+\frac{x_{1 i}-\mu_{1}}{\sigma_{1}}\right)
\end{aligned}
$$


Direct maximum likelihood estimates of the parameters based on $\left(X_{1 i}, X_{2 i}\right): i=$ 1(1) $n$ may not be simple. We implement EM algorithm first given some $\mu_{1}, \mu_{2}, \sigma_{1}$ and $\sigma_{2}$. It requires identification of some missing structure within the problem.

We do not know $X_{1}$ is $\sigma_{1} U_{0}+\mu_{1}$ or $U_{1}$ and We do not know $X_{2}$ is $\sigma_{2} U_{0}+\mu_{2}$ or $U_{2}$. So we introduce two new random variables $\left(\Delta_{1} ; \Delta_{2}\right)$ as

$$
\Delta_{1}= \begin{cases}0 & \text { if } X_{1}=\sigma_{1} U_{0}+\mu_{1} \\ 1 & \text { if } X_{1}=U_{1}\end{cases}
$$

and

$$
\Delta_{2}= \begin{cases}0 & \text { if } X_{2}=\sigma_{2} U_{0}+\mu_{2} \\ 2 & \text { if } X_{2}=U_{2}\end{cases}
$$

The $\Delta_{1}$ and $\Delta_{2}$ are the missing values of the E-M algorithm. To calculate the E-step we need the conditional distribution of $\Delta_{1}$ and $\Delta_{2}$.

Using the definition of $X_{1}, X_{2}, \Delta_{1}$ and $\Delta_{2}$ we have :

$\triangleright$ For group $I_{0}$, both $\Delta_{1}$ and $\Delta_{2}$ are known,

$$
\Delta_{1}=\Delta_{2}=0
$$

$\triangleright$ For group $I_{1}, \Delta_{1}$ is known, $\Delta_{2}$ is unknown,

$$
\Delta_{1}=1, \Delta_{2}=0 \text { or } 2
$$

Therefore we need to find out $u_{1}=P\left(\Delta_{2}=0 \mid I_{1}\right)$ and $u_{2}=P\left(\Delta_{2}=2 \mid I_{1}\right)$

$\triangleright$ For group $I_{2}, \Delta_{2}$ is known, $\Delta_{1}$ is unknown,

$$
\Delta_{1}=0 \text { or } 1, \Delta_{2}=2
$$

Moreover we need $w_{1}=P\left(\Delta_{1}=0 \mid I_{2}\right)$ and $w_{2}=P\left(\Delta_{1}=1 \mid I_{2}\right)$

Since, each posterior probability corresponds to one of the ordering from Table1 , we calculate $u_{1}, u_{2}, w_{1}, w_{2}$ using the probability of appropriate ordering, where $U_{1}^{*}=\frac{\left(U_{1}-\mu_{1}\right)}{\sigma_{1}}, U_{2}^{*}=\frac{\left(U_{2}-\mu_{2}\right)}{\sigma_{2}}$.

\begin{tabular}{|c|c|r|}
\hline Ordering & $\left(X_{1}, X_{2}\right)$ & Group \\
\hline$U_{0}<U_{1}^{*}<U_{2}^{*}$ & $\left(U_{0}, U_{0}\right)$ & $I_{0}$ \\
$U_{0}<U_{2}^{*}<U_{1}^{*}$ & $\left(U_{0}, U_{0}\right)$ & $I_{0}$ \\
$U_{1}^{*}<U_{0}<U_{2}^{*}$ & $\left(U_{1}^{*}, U_{0}\right)$ & $I_{1}$ \\
$U_{1}^{*}<U_{2}^{*}<U_{0}$ & $\left(U_{1}^{*}, U_{2}^{*}\right)$ & $I_{1}$ \\
$U_{2}^{*}<U_{0}<U_{1}^{*}$ & $\left(U_{0}, U_{2}^{*}\right)$ & $I_{2}$ \\
$U_{2}^{*}<U_{1}^{*}<U_{0}$ & $\left(U_{1}^{*}, U_{2}^{*}\right)$ & $I_{2}$ \\
\hline
\end{tabular}

Table 1 Groups and corresponding orderings of hidden random variables $U_{0}, U_{1}^{*}$ and $U_{2}^{*}$

We have the following expressions for $u_{1}, u_{2}, w_{1}, w_{2}$

$$
u_{1}=\frac{P\left(U_{1}^{*}<U_{0}<U_{2}^{*}\right)}{P\left(U_{1}^{*}<U_{0}<U_{2}^{*}\right)+P\left(U_{1}^{*}<U_{2}^{*}<U_{0}\right)}
$$


Now we have

$$
\begin{aligned}
& u_{2}=\frac{P\left(U_{1}^{*}<U_{2}^{*}<U_{0}\right)}{P\left(U_{1}^{*}<U_{0}<U_{2}^{*}\right)+P\left(U_{1}^{*}<U_{2}^{*}<U_{0}\right)} \\
& w_{1}=\frac{P\left(U_{2}^{*}<U_{0}<U_{1}^{*}\right)}{P\left(U_{2}^{*}<U_{0}<U_{1}^{*}\right)+P\left(U_{2}^{*}<U_{1}^{*}<U_{0}\right)} \\
& w_{2}=\frac{P\left(U_{2}^{*}<U_{1}^{*}<U_{0}\right)}{P\left(U_{2}^{*}<U_{0}<U_{1}^{*}\right)+P\left(U_{2}^{*}<U_{1}^{*}<U_{0}\right)}
\end{aligned}
$$

$$
\begin{aligned}
P\left(U_{1}^{*}<U_{0}<U_{2}^{*}\right) & =\int_{0}^{\infty}\left[1-(1+x)^{-\alpha_{1}}\right] \alpha_{0}(1+x)^{-\left(\alpha_{0}+1\right)}(1+x)^{-\left(\alpha_{2}\right)} \\
& =\alpha_{0} \int_{0}^{\infty}(1+x)^{-\left(\alpha_{0}+\alpha_{2}+1\right)}-(1+x)^{-\left(\alpha_{0}+\alpha_{1}+\alpha_{2}+1\right)} \\
& =\frac{\alpha_{0} \alpha_{1}}{\left(\alpha_{0}+\alpha_{2}+1\right)\left(\alpha_{0}+\alpha_{1}+\alpha_{2}+1\right)}
\end{aligned}
$$

Using this above result we evaluate the other probabilites to get values of $u_{1}, u_{2}, w_{1}, w_{2}$ as : $u_{1}=\frac{\alpha_{0}}{\alpha_{0}+\alpha_{2}}$ and $u_{2}=\frac{\alpha_{2}}{\alpha_{0}+\alpha_{2}} w_{1}=\frac{\alpha_{0}}{\alpha_{0}+\alpha_{1}}$ and $w_{2}=\frac{\alpha_{1}}{\alpha_{0}+\alpha_{1}}$

\subsection{Pseudo-likelihood expression}

We define $n_{0}, n_{1}, n_{2}$ as : $n_{0}=\left|I_{0}\right|, n_{1}=\left|I_{1}\right|, n_{2}=\left|I_{2}\right|$ where $\left|I_{j}\right|$ for $j=0,1,2$ denotes the number of elements in the set $I_{j}$. Now the pseudo log-likelihood can be written down as

$$
\begin{aligned}
Q & =L\left(\alpha_{0}, \alpha_{1}, \alpha_{2}\right) \\
& =-\alpha_{0}\left(\sum_{i \in I_{0}} \ln \left(1+x_{i}\right)+\sum_{i \in I_{2}} \ln \left(1+x_{1 i}\right)+\sum_{i \in I_{1}} \ln \left(1+x_{2 i}\right)\right) \\
& +\left(n_{0}+u_{1} n_{1}+w_{1} n_{2}\right) \ln \alpha_{0}-\alpha_{1}\left(\sum_{i \in I_{0}} \ln \left(1+x_{i}\right)+\sum_{i \in I_{1} \cup I_{2}} \ln \left(1+x_{1 i}\right)\right) \\
& +\left(n_{1}+w_{2} n_{2}\right) \ln \alpha_{1}-\alpha_{2}\left(\sum_{i \in I_{0}} \ln \left(1+x_{i}\right)+\sum_{i \in I_{1} \cup I_{2}} \ln \left(1+x_{2 i}\right)\right) \\
& +\left(n_{2}+u_{2} n_{1}\right) \ln \alpha_{2}
\end{aligned}
$$

Therefore M-step involves maximizing (1) with respect to $\alpha_{0}, \alpha_{1}, \alpha_{2}$ at

$$
\begin{gathered}
\hat{\alpha}_{0}^{(t+1)}=\frac{n_{0}+u_{1}^{(t)} n_{1}+w_{1}^{(t)} n_{2}}{\sum_{i \in I_{0}} \ln \left(1+x_{i}\right)+\sum_{i \in I_{2}} \ln \left(1+x_{1 i}\right)+\sum_{i \in I_{1}} \ln \left(1+x_{2 i}\right)} \\
\hat{\alpha}_{1}^{(t+1)}=\frac{\left(n_{1}+w_{2}^{(t)} n_{2}\right)}{\sum_{i \in I_{0}} \ln \left(1+x_{i}\right)+\sum_{i \in\left(I_{1} \cup I_{2}\right)} \ln \left(1+x_{1 i}\right)} \\
\hat{\alpha}_{2}^{(t+1)}=\frac{n_{2}+u_{2}^{(t)} n_{1}}{\left(\sum_{i \in I_{0}} \ln \left(1+x_{i}\right)+\sum_{i \in\left(I_{1} \cup I_{2}\right)} \ln \left(1+x_{2 i}\right)\right)}
\end{gathered}
$$

Therefore the algorithm can be given as 


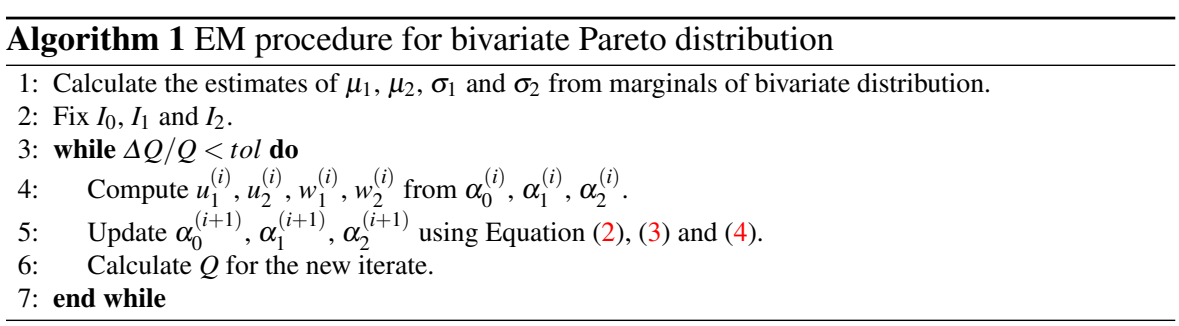

\subsection{Algorithm-2 : Modified Algorithm 1}

The above algorithm does not work even if all the $I_{0}, I_{1}$ and $I_{2}$ are non-empty. In our previous approach we calculate the estimates of location and scale parameters and construct the above three sets based on the transformed variables using those estimated location and scale parameters. Therefore it is high likely that there will be almost no element in $I_{0}$ for most of the generated sample. Consequently it should provide the estimate of $\alpha_{0}$ as zero most of the time.

We propose to make few modifications in the above EM algorithm. Since we observe the normalized data with respect to the estimated location and scale parameter, the transformation is not going to provide the distribution of normalized data exactly as Pareto with location zero and scale one. The transformation rather form some distribution close to Pareto with location zero and scale one. It is very difficult to know the exact distribution. Approximately even if we assume some Pareto with location parameter near zero and scale parameter near one, calculation of probability that an observation will come from any one of $I_{0}, I_{1}$ or $I_{2}$, becomes another problem.

To avoid such instances, we assume that information of all $n_{0}, n_{1}$ and $n_{2}$ are missing because of small perturbation inherited by estimated location and scale parameters. In ideal situation we should get EM algorithm steps as described in 3. Since we have now three more latent information $n_{0}, n_{1}$ and $n_{2}$, we try to approximate them and incorporate in the original EM algorithm.

It is clear that $\left(n_{0}, n_{1}, n_{2}\right)$ will jointly follow multinomial distribution with parameters $n=n_{0}+n_{1}+n_{2}$ and $\left(\frac{\alpha_{0}}{\alpha_{0}+\alpha_{1}+\alpha_{2}}, \frac{\alpha_{1}}{\alpha_{0}+\alpha_{1}+\alpha_{2}}, \frac{\alpha_{2}}{\alpha_{0}+\alpha_{1}+\alpha_{2}}\right)$.

We approximate $\tilde{n_{i}}=n \cdot \frac{\alpha_{i}}{\alpha_{0}+\alpha_{1}+\alpha_{2}}, \quad i=0,1,2$.

Therefore M-step involves maximizing (1) with respect to $\alpha_{0}, \alpha_{1}, \alpha_{2}$ at

$$
\begin{gathered}
\hat{\alpha}_{0}^{(t+1)}=\frac{\tilde{n_{0}}+u_{1}^{(t)} \tilde{n_{1}}+w_{1}^{(t)} \tilde{n_{2}}}{\sum_{i \in I_{0}} \ln \left(1+x_{i}\right)+\sum_{i \in I_{2}} \ln \left(1+x_{1 i}\right)+\sum_{i \in I_{1}} \ln \left(1+x_{2 i}\right)} . \\
\hat{\alpha}_{1}^{(t+1)}=\frac{\left(\tilde{n_{1}}+w_{2}^{(t)} \tilde{n_{2}}\right)}{\sum_{i \in I_{0}} \ln \left(1+x_{i}\right)+\sum_{i \in\left(I_{1} \cup I_{2}\right)} \ln \left(1+x_{1 i}\right)} \\
\hat{\alpha}_{2}^{(t+1)}=\frac{\tilde{n_{2}}+u_{2}^{(t)} \tilde{n_{1}}}{\left(\sum_{i \in I_{0}} \ln \left(1+x_{i}\right)+\sum_{i \in\left(I_{1} \cup I_{2}\right)} \ln \left(1+x_{2 i}\right)\right)}
\end{gathered}
$$

Finally modified algorithm would be 


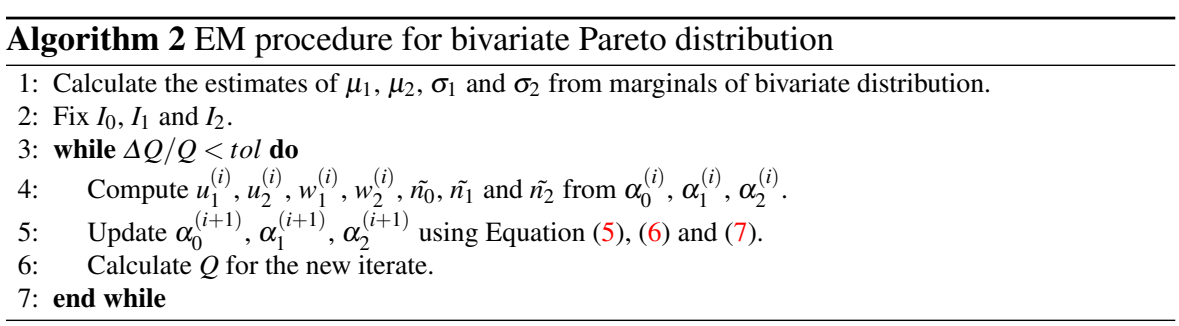

\subsection{Algorithm-3 : Modified Algorithm 2}

In this case we update $\sigma_{1}$ and $\sigma_{2}$ along with EM iterations similar to what Asimit et al. has done in their paper. But the main difference of our update is that it is based on one step ahead gradient descent instead of fixed point iteration on scale parameters. Surely gradient descent with respect to bivariate likelihood won't work as bivariate likelihood is a discontinuous function with respect to location and scale parameters. Therefore we estimate scale parameters using density of marginals. At every iteration we use one step ahead gradient descend of $\sigma_{1}$ and $\sigma_{2}$ based on likelihood of its marginal density combined with usual EM steps for other parameters to solve the problem. The idea is similar to stochastic gradient descend. We begin the algorithm estimating the sets $I_{0}, I_{1}$ and $I_{2}$ which involves location and scale parameters. Given location and scale parameters, we can update previous EM steps of $\alpha_{0}, \alpha_{1}$ and $\alpha_{2}$. EM steps will ensure to take the correct direction of $\alpha_{0}, \alpha_{1}$ and $\alpha_{2}$ starting from any values of this three parameters and also it will enable gradient descend steps of $\sigma_{1}$ and $\sigma_{2}$ to converge faster starting from any values.

This algorithm works even for moderate sample sizes. However it takes lot of time to converge or roam around the actual value for some really bad sample. We calculate the estimates both with full iterations until convergence and with at most 2000 iterations. We observe MSEs and the number of iterations in both the cases. We expect that within 2000 iterations estimated points should be sufficiently closer to the actual value or it should take some value which can be good estimate for starting point of some other optimization algorithms.

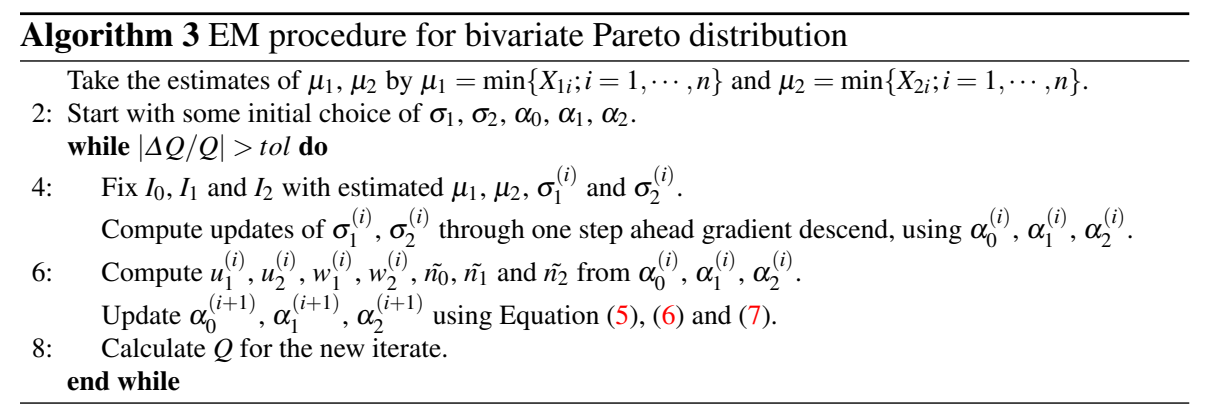




\subsection{Algorithm-4 : Modified Algorithm 3}

We can make more variations on it. This is similar to what Asimit et al. has done in their paper. At each iteration we update $\sigma_{1}$ and $\sigma_{2}$ from the marginal density using one step ahead fixed point iteration instead of Gradient decend algorithm and carry on the same EM algorithm. However approach of Asimit et al is different, therefore EM steps are also differing.

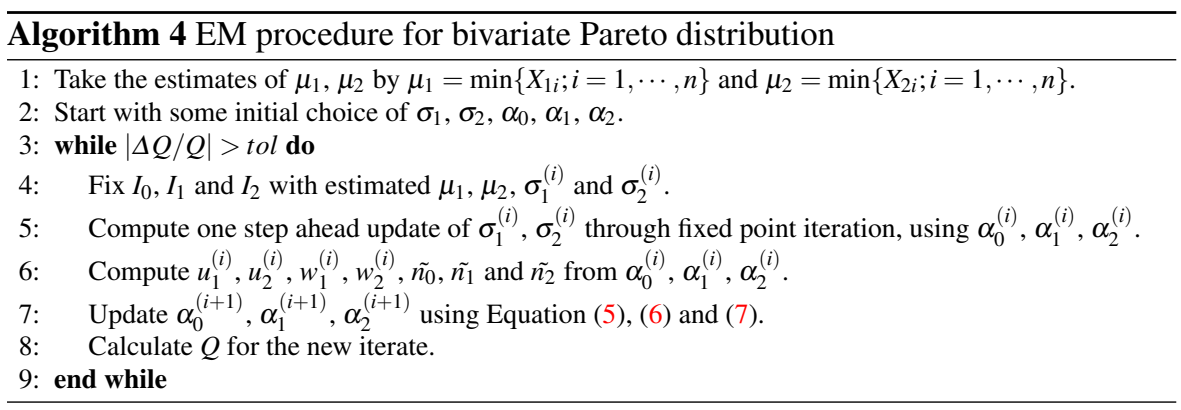

\subsection{Algorithm-5 : Modified Algorithm 4}

This variation is of two fold. We update $\sigma_{1}$ and $\sigma_{2}$ from the marginal density until convergence using fixed point iteration instead of Gradient decent algorithm and carry forward the same EM algorithm. However at the time of using EM iterations, we update the $\sigma_{1}$ and $\sigma_{2}$ using one step ahead fixed point iteration along with other parameters. The detailed algorithmic steps are shown below.

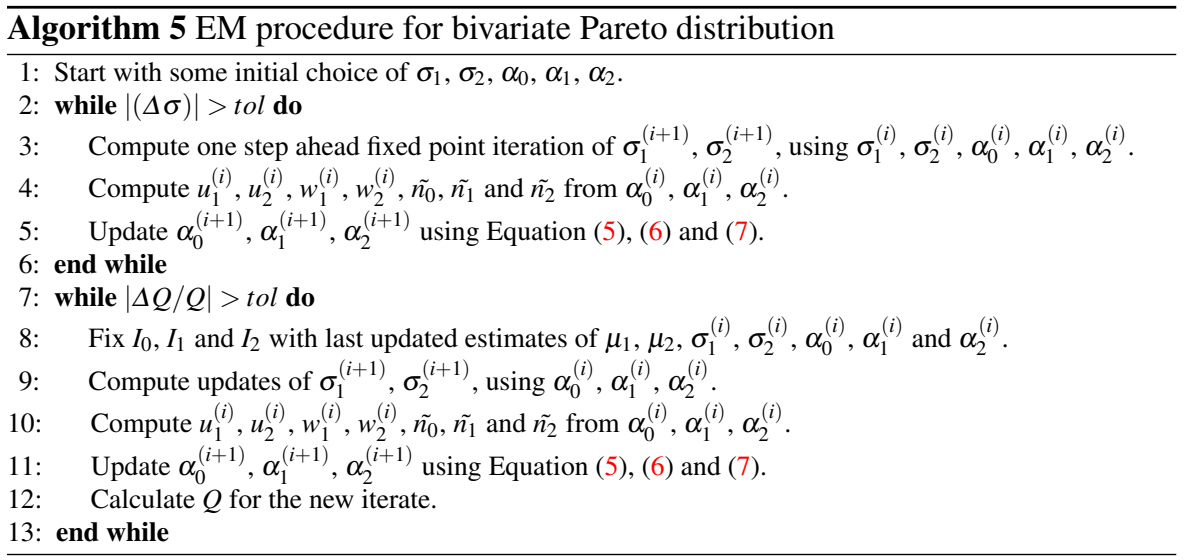




\section{Numerical Results}

We use package R 3.2.3 to perform the estimation procedure. All the programs will be available from author on request. First we take four different sets of parameters and observe the average iteration calculated over different sample sizes. We take our sample size as $n=150,250,350,450$. We can find the results in Table- 2 based on 1000 replications. Tolerance limit (denoted as "tol") of stopping criteria is taken as 0.00001 . We have used stopping criteria as absolute value of likelihood changes with respect to previous Qseudo-likelihood at each iteration. Results shown in Table-2 provide average iteration by six different approaches. They are (a) Modified Approach 1 (b) Modified Approach 2 which we truncate after 2000 iterations and denote this as Modified Approach 2 (T) (c) Modified Approach 2 without truncating the iteration and denote this as Modified Approach 2 (WT) (d) Modified Approach 3 (e) Modified Approach 4 (f) Asimit's approach. We choose four different sets of parameters to compare the results. These are $\mu_{1}=0, \mu_{2}=0, \sigma_{1}=1, \sigma_{2}=0.5, \alpha_{0}=1, \alpha_{1}=$ $0.3, \alpha_{2}=1.4 ; \quad \mu_{1}=1, \mu_{2}=2, \sigma_{1}=0.4, \sigma_{2}=0.5, \alpha_{0}=2, \alpha_{1}=1.2, \alpha_{2}=1.4 ; \quad \mu_{1}=$ $0, \mu_{2}=0, \sigma_{1}=1.4, \sigma_{2}=0.5, \alpha_{0}=1, \alpha_{1}=1, \alpha_{2}=1.4 ; \quad \mu_{1}=0, \mu_{2}=0, \sigma_{1}=$ $1.4, \sigma_{2}=0.5, \alpha_{0}=2, \alpha_{1}=0.4, \alpha_{2}=0.5$. The proposed EM algorithms work for any initial value. However estimates of $\mu_{1}$ and $\mu_{2}$ are always $\hat{\mu}_{1}=\min \left\{X_{1 i} ; 1, \cdots, n\right\}$ and $\hat{\mu}_{2}=\min \left\{X_{2 i} ; 1, \cdots, n\right\}$ respectively. Original paper of Asimit et al. uses different stopping criteria and results are provided for large sample sizes. To compare our results, we keep stopping criteria and sample size same across all algorithms.

Average estimates are provided in Table- 3 when samples are simulated from bivariate pareto with parameters $\mu_{1}=0, \mu_{2}=0, \sigma_{1}=1, \sigma_{2}=0.5, \alpha_{0}=1, \alpha_{1}=0.3, \alpha_{2}=$ 1.4. Results of average estimates are shown only by two best approaches (best in the sense of mininum average iteration) i.e. Modified Approach 1 and Modified Approach 4. Table-4 and Table-5 show the MSEs for all procedures where samples are generated from the following parameter sets : $\mu_{1}=0, \mu_{2}=0, \sigma_{1}=1, \sigma_{2}=0.5, \alpha_{0}=$ $1, \alpha_{1}=0.3, \alpha_{2}=1.4$. MSE is an important criteria for selecting the best algorithm. Therefore MSEs are calculated for all the procedures. We also calculate parametric bootstrap confidence interval. We simulate 1000 samples and estimate of the parameters based on the simulated samples. We use them to get the $95 \%$ confidence interval by calculating 0.025 and 0.975 sample quantile points of estimated parameters. Table- 6 to Table-10 carry the information related to parametric bootstrap confidence intervals.

Important Comments :

1. Mean square error for $\alpha_{2}$ is little higher for all methods.

2. MSEs are more or less same for all methods. In the above result we see Modified Approach 1 provides minimum MSE among all procedures. For large sample size Modified Approach 1 comes out as winner for any chosen parameter sets. Performance of Modified Approach 2 with truncation is also worth mentioning, as it provides second best performance with respect to minimum MSE.

3. Modified Approach 4 is the best among other approaches in terms of average iteration. Modified Approach 1 appears to be the second best performer. How- 
ever average iteration for Modified Approach 2 with truncation is much higher as compared to Modified Approach 1 and 4.

4. As expected, average iteration is very high in case of Modified Approach 2 (WT).

5. If we compare the algorithms based on minimum MSE and average iteration together, Modified approach 1 stands either winner or closer to the winner.

\begin{tabular}{|c|c|c|c|c|c|c|}
\hline parameter set & & $\mathrm{n}$ & 150 & 250 & 350 & 450 \\
\hline $\begin{array}{c}\mu_{1}=0, \mu_{2}=0 \\
\sigma_{1}=1 \sigma_{2}=0.5 \\
\alpha_{0}=1, \alpha_{1}=0.3 \\
\alpha_{2}=1.4\end{array}$ & $\begin{array}{c}\text { The average } \\
\text { number } \\
\text { of Iterations } \\
\text { (AI) }\end{array}$ & $\begin{array}{c}\text { Modified } \\
\text { Approach } 1 \\
\text { Modified } \\
\text { Approach 2 (WT) } \\
\text { Modified } \\
\text { Approach 2 (T) } \\
\text { Modified } \\
\text { Approach 3 } \\
\text { Modified } \\
\text { Approach 4 } \\
\text { Asimit's Approach }\end{array}$ & $\begin{array}{c}3402 \\
1771 \\
266 \\
191 \\
1002\end{array}$ & $\begin{array}{c}2163 \\
1631 \\
249 \\
162 \\
945\end{array}$ & $\begin{array}{c}1542 \\
1414 \\
238 \\
151 \\
909\end{array}$ & $\begin{array}{r}1220 \\
1194 \\
234 \\
147 \\
902\end{array}$ \\
\hline $\begin{array}{c}\mu_{1}=1, \mu_{2}=2 \\
\sigma_{1}=0.4 \sigma_{2}=0.5 \\
\alpha_{0}=2, \alpha_{1}=1.2, \\
\alpha_{2}=1.4\end{array}$ & $\begin{array}{c}\text { The average } \\
\text { number } \\
\text { of Iterations } \\
\text { (AI) }\end{array}$ & $\begin{array}{c}\text { Modified } \\
\text { Approach } 1 \\
\text { Modified } \\
\text { Approach 2 (WT) } \\
\text { Modified } \\
\text { Approach 2 (T) } \\
\text { Modified } \\
\text { Approach } 3 \\
\text { Modified } \\
\text { Approach 4 } \\
\text { Asimit's Approach }\end{array}$ & $\begin{array}{r}174 \\
7734 \\
1704 \\
764 \\
243 \\
2345\end{array}$ & $\begin{array}{r}4137 \\
1553 \\
551 \\
185 \\
1747\end{array}$ & $\begin{array}{r}2428 \\
1378 \\
458 \\
153 \\
1464\end{array}$ & $\begin{array}{r}1633 \\
1184 \\
424 \\
128 \\
1309\end{array}$ \\
\hline $\begin{array}{c}\mu_{1}=0, \mu_{2}=0 \\
\sigma_{1}=1.4 \sigma_{2}=0.5 \\
\alpha_{0}=1, \alpha_{1}=1, \\
\alpha_{2}=1.4\end{array}$ & $\begin{array}{c}\text { The average } \\
\text { number } \\
\text { of Iterations } \\
\text { (AI) }\end{array}$ & $\begin{array}{c}\text { Modified } \\
\text { Approach 1 } \\
\text { Modified } \\
\text { Approach 2 (WT) } \\
\text { Modified } \\
\text { Approach 2 (T) } \\
\text { Modified } \\
\text { Approach 3 } \\
\text { Modified } \\
\text { Approach 4 } \\
\text { Asimit's Approach }\end{array}$ & $\begin{array}{r}168 \\
7230 \\
1617 \\
265 \\
123 \\
897\end{array}$ & $\begin{array}{r}155 \\
4372 \\
1738 \\
237 \\
101 \\
547\end{array}$ & $\begin{array}{r}150 \\
3077 \\
1732 \\
220 \\
91 \\
484\end{array}$ & $\begin{array}{c}147 \\
2423 \\
1697 \\
209 \\
83\end{array}$ \\
\hline $\begin{array}{c}\mu_{1}=0, \mu_{2}=0 \\
\sigma_{1}=1.4, \sigma_{2}=0.5 \\
\alpha_{0}=2, \alpha_{1}=0.4, \\
\alpha_{2}=0.5\end{array}$ & $\begin{array}{c}\text { The average } \\
\text { number } \\
\text { of Iterations } \\
\text { (AI) }\end{array}$ & $\begin{array}{c}\text { Modified } \\
\text { Approach 1 } \\
\text { Modified } \\
\text { Approach 2 (WT) } \\
\text { Modified } \\
\text { Approach 2 (T) } \\
\text { Modified } \\
\text { Approach 3 } \\
\text { Modified } \\
\text { Approach 4 } \\
\text { Asimit's Approach }\end{array}$ & $\begin{array}{r}138 \\
2172 \\
397 \\
330 \\
117 \\
1145\end{array}$ & $\begin{array}{l}2707 \\
591 \\
261 \\
100 \\
977\end{array}$ & $\begin{array}{c}2237 \\
773 \\
238 \\
96 \\
919\end{array}$ & $\begin{array}{c}1821 \\
881 \\
219 \\
95 \\
875\end{array}$ \\
\hline
\end{tabular}

Table 2 Average number of iterations (AI) by six approaches: (a) Modified Approach 1 (b) Modified Approach 2 (WT) (c) Modified Approach 2 (T) (d) Modified Approach 3 (e) Modified Approach 4 (f) Asimit's Approach 


\begin{tabular}{|c|c|c|c|c|c|c|c|c|}
\hline $\mathrm{n}$ & parameters & $\mu_{1}$ & $\mu_{2}$ & $\sigma_{1}$ & $\sigma_{2}$ & $\alpha_{0}$ & $\alpha_{1}$ & $\alpha_{2}$ \\
\hline \multirow[t]{2}{*}{150} & $\begin{array}{l}\text { Average (Mod. 1) } \\
\text { estimates } \\
\text { (AE) }\end{array}$ & 0.0056 & 0.0014 & 0.9031 & 0.5154 & 0.8993 & 0.3379 & 1.5660 \\
\hline & $\begin{array}{c}\text { Average (Mod. 4) } \\
\text { estimates } \\
\text { (AE) }\end{array}$ & 0.0056 & 0.0014 & 1.0521 & 0.5755 & 0.9372 & 0.4122 & 1.7416 \\
\hline \multirow[t]{2}{*}{250} & $\begin{array}{c}\text { Average (Mod. 1) } \\
\text { Estimates } \\
\text { (AE) }\end{array}$ & 0.0031 & 0.0008 & 0.8943 & 0.4989 & 0.9013 & 0.3226 & 1.5046 \\
\hline & $\begin{array}{c}\text { Average (Mod. 4) } \\
\text { estimates } \\
\text { (AE) }\end{array}$ & 0.0031 & 0.0008 & 1.0386 & 0.5457 & 0.9408 & 0.3909 & 1.6292 \\
\hline \multirow[t]{2}{*}{350} & $\begin{array}{l}\text { Average (Mod. 1) } \\
\text { Estimates } \\
\text { (AE) }\end{array}$ & 0.0022 & 0.0006 & 0.8854 & 0.4878 & 0.9086 & 0.3107 & 1.4550 \\
\hline & $\begin{array}{c}\text { Average (Mod. 4) } \\
\text { estimates } \\
\text { (AE) }\end{array}$ & 0.0022 & 0.0006 & 1.0252 & 0.5283 & 0.9497 & 0.3745 & 1.5543 \\
\hline \multirow[t]{2}{*}{450} & $\begin{array}{l}\text { Average (Mod. 1) } \\
\text { Estimates } \\
\text { (AE) }\end{array}$ & 0.0017 & 0.0005 & 0.8719 & 0.4799 & 0.9099 & 0.2955 & 1.4270 \\
\hline & $\begin{array}{c}\text { Average (Mod. 4) } \\
\text { estimates } \\
\text { (AE) }\end{array}$ & 0.0017 & 0.0005 & 1.0077 & 0.5187 & 0.9492 & 0.3586 & 1.5221 \\
\hline
\end{tabular}

Table 3 The average estimates (AE) for $\mu_{1}=0, \mu_{2}=0, \sigma_{1}=1, \sigma_{2}=0.5, \alpha_{0}=1, \alpha_{1}=0.3$ and $\alpha_{2}=1.4$ through two best approches i.e. Modified Approach 1 and Modified Approach 4

\begin{tabular}{|c|c|c|c|c|}
\hline & parameters & $\mu_{1}$ & $\mu_{2}$ & $\sigma_{1}$ \\
\hline $\mathrm{n}=150$ & $\begin{array}{c}\text { MSE (Mod. 1) } \\
\text { MSE (Mod. } 2 \text { (T)) } \\
\text { MSE (Mod. } 2 \text { (WT)) } \\
\text { MSE (Mod. 3) } \\
\text { MSE (Mod. 4) } \\
\text { MSE (Asimit) }\end{array}$ & $\begin{array}{c}6.14 \mathrm{e}-05 \\
0.0000614 \\
0.0000614 \\
0.0000614 \\
0.0000614 \\
0.0000614\end{array}$ & $\begin{array}{c}3.69 \mathrm{e}-06 \\
0.0000037 \\
0.0000370 \\
0.0000037 \\
0.0000037 \\
0.0000037\end{array}$ & $\begin{array}{l}0.0832 \\
0.0543 \\
0.1043 \\
0.1216 \\
0.1179 \\
0.1218\end{array}$ \\
\hline $\begin{array}{c}\mathrm{n}=150 \\
\text { MSE (Mod. 1) } \\
\text { MSE (Mod. 2 (T)) } \\
\text { MSE (Mod. 2 (WT)) } \\
\text { MSE (Mod. 3) } \\
\text { MSE (Mod. 4) } \\
\text { MSE (Asimit) }\end{array}$ & $\begin{array}{c}\sigma_{2} \\
0.0477 \\
0.0368 \\
0.0682 \\
0.1028 \\
0.1090 \\
0.1011 \\
\end{array}$ & $\begin{array}{c}\alpha_{0} \\
0.0402 \\
0.0386 \\
0.0433 \\
0.0439 \\
0.0429 \\
0.0433 \\
\end{array}$ & $\begin{array}{c}\alpha_{1} \\
0.0375 \\
0.0342 \\
0.0609 \\
0.0737 \\
0.0691 \\
0.0726\end{array}$ & $\begin{array}{c}\alpha_{2} \\
0.5178 \\
0.4154 \\
0.7969 \\
1.2035 \\
1.2536 \\
1.1860\end{array}$ \\
\hline & parameters & $\mu_{1}$ & $\mu_{2}$ & $\sigma_{1}$ \\
\hline $\begin{array}{c}\mathrm{n}=250 \\
\text { MSE (Mod. 1) } \\
\text { MSE (Mod. 2 (T)) } \\
\text { MSE (Mod. 2 (WT)) } \\
\text { MSE (Mod. 3) } \\
\text { MSE (Mod. 4) } \\
\text { MSE (Asimit) }\end{array}$ & $\begin{array}{c}\text { MSE (Mod. 1) } \\
\text { MSE (Mod. 2 (T)) } \\
\text { MSE (Mod. 2 (WT)) } \\
\text { MSE (Mod. 3) } \\
\text { MSE (Mod. 4) } \\
\text { MSE (Asimit) } \\
\sigma_{2} \\
0.0278 \\
0.0368 \\
0.0410 \\
0.0461 \\
0.0460 \\
0.0462\end{array}$ & $\begin{array}{c}0.000020 \\
0.0000614 \\
0.0000198 \\
0.00001978 \\
0.00001978 \\
0.00001978 \\
\alpha_{0} \\
0.0296 \\
0.0386 \\
0.0294 \\
0.0296 \\
0.0289 \\
0.0292\end{array}$ & $\begin{array}{c}1.4757 \mathrm{e}-06 \\
0.00000370 \\
0.0000014 \\
0.00000147 \\
0.00000147 \\
0.00000148 \\
\alpha_{1} \\
0.0205 \\
0.0342 \\
0.0386 \\
0.0431 \\
0.0401 \\
0.0427\end{array}$ & $\begin{array}{l}0.0581 \\
0.0543 \\
0.0682 \\
0.0745 \\
0.0724 \\
0.0746 \\
\alpha_{2} \\
0.2817 \\
0.4153 \\
0.4441 \\
0.5033 \\
0.4978 \\
0.5638\end{array}$ \\
\hline
\end{tabular}

Table 4 Mean Square Error (MSE) through all approaches when samples are generated from $\mu_{1}=0$, $\mu_{2}=0, \sigma_{1}=1, \sigma_{2}=0.5, \alpha_{0}=1, \alpha_{1}=0.3$ and $\alpha_{2}=1.4$ 


\begin{tabular}{|c|c|c|c|c|}
\hline & parameters & $\mu_{1}$ & $\mu_{2}$ & $\sigma_{1}$ \\
\hline $\mathrm{n}=350$ & MSE (Mod. 1) & $9.236 \mathrm{e}-06$ & $6.992 \mathrm{e}-07$ & 0.0427 \\
& MSE (Mod. 2 (T)) & 0.00000197 & 0.000000699 & 0.0389 \\
& MSE (Mod. 2 (WT)) & 0.00000924 & 0.000000699 & 0.0428 \\
& MSE (Mod. 3) & 0.00000923 & 0.000000699 & 0.0456 \\
& MSE (Mod. 4) & 0.00000924 & 0.000000699 & 0.0440 \\
MSE (Asimit) & 0.00000923 & 0.000000699 & 0.0454 \\
n=350 & $\sigma_{2}$ & $\alpha_{0}$ & $\alpha_{1}$ & $\alpha_{2}$ \\
MSE (Mod. 1) & 0.0155 & 0.0211 & 0.0143 & 0.1475 \\
MSE (Mod. 2 (T)) & 0.02001 & 0.01907 & 0.02573 & 0.2068 \\
MSE (Mod. 2(WT)) & 0.0213 & 0.0192 & 0.0280 & 0.2204 \\
MSE (Mod. 3) & 0.0221 & 0.0194 & 0.0302 & 0.2290 \\
MSE (Mod. 4) & 0.0220 & 0.0188 & 0.0275 & 0.2252 \\
MSE (Asimit) & 0.0222 & 0.0194 & 0.0301 & 0.2290 \\
\hline & parameters & $\mu_{1}$ & $\mu_{2}$ & $\sigma_{1}$ \\
& & & & \\
\hline $\mathrm{n}=450$ & MSE (Mod. 1) & $5.589 \mathrm{e}-06$ & $4.398 \mathrm{e}-07$ & 0.0366 \\
& MSE (Mod. 2 (T)) & 0.0000055 & 0.000000440 & 0.0283 \\
& MSE (Mod. 2 (WT)) & 0.00000559 & 0.00000044 & 0.0288 \\
& MSE (Mod. 3) & 0.00000559 & 0.00000047 & 0.03008 \\
$\mathrm{n}=450$ & MSE (Mod. 4) & 0.00000558 & 0.000000439 & 0.0292 \\
MSE (Mod. 1) & MSE (Asimit) & 0.00000559 & 0.000000440 & 0.0300 \\
MSE (Mod. 2 (T)) & $\sigma_{2}$ & $\alpha_{0}$ & $\alpha_{1}$ & $\alpha_{2}$ \\
MSE (Mod. 2 (WT)) & 0.0107 & 0.0176 & 0.0097 & 0.1140 \\
MSE (Mod. 3) & 0.0143 & 0.015 & 0.0181 & 0.1655 \\
MSE (Mod. 4) & 0.0147 & 0.0151 & 0.0184 & 0.1717 \\
MSE (Asimit) & 0.0151 & 0.0152 & 0.0196 & 0.1759 \\
& 0.0150 & 0.0148 & 0.0179 & 0.1736 \\
& 0.0151 & 0.0151 & 0.0195 & 0.1756 \\
\hline
\end{tabular}

Table 5 Mean Square Error (MSE) through all approaches when samples are generated from $\mu_{1}=0$, $\mu_{2}=0, \sigma_{1}=1, \sigma_{2}=0.5, \alpha_{0}=1, \alpha_{1}=0.3$ and $\alpha_{2}=1.4$

\begin{tabular}{|c|c|c|c|c|}
\hline $\mathrm{n}$ & 150 & 250 & 350 & 450 \\
parameters & & & & \\
\hline$\mu_{1}$ & {$[0.0001,0.0193]$} & {$[0.000077,0.0125]$} & {$[0.00007,0.0079]$} & {$[0.00004,0.0062]$} \\
$\mu_{2}$ & {$[0.000034,0.0050]$} & {$[0.000027,0.0034]$} & {$[0.00002,0.0022]$} & {$[0.00001,0.0017]$} \\
$\sigma_{1}$ & {$[0.5112,1.4976]$} & {$[0.5513,1.3754]$} & {$[0.6016,1.2959]$} & {$[0.6257,1.1722]$} \\
$\sigma_{2}$ & {$[0.2361,1.0501]$} & {$[0.2796,0.9011]$} & {$[0.3067,0.7946]$} & {$[0.3183,0.7207]$} \\
$\alpha_{0}$ & {$[0.6103,1.2854]$} & {$[0.6682,1.1940]$} & {$[0.7004,1.1586]$} & {$[0.7373,1.1197]$} \\
$\alpha_{1}$ & {$[0.0974,0.8052]$} & {$[0.1172,0.6801]$} & {$[0.1206,0.5963]$} & {$[0.1241,0.5377]$} \\
$\alpha_{2}$ & {$[0.7692,3.3941]$} & {$[0.8701,2.8723]$} & {$[0.9447,2.3398]$} & {$[0.9772,2.2408]$} \\
\hline
\end{tabular}

Table 6 Parametric Bootstrap confidence interval for $\mu_{1}=0, \mu_{2}=0, \sigma_{1}=1, \sigma_{2}=0.5, \alpha_{0}=1, \alpha_{1}=0.3$ and $\alpha_{2}=1.4$ in Modified Approach 1

\section{Data Analysis}

We analyze a data set on the indemnity payments (Loss) and allocated loss adjustment expense (ALAE) relating to 1500 general liability claims from insurance companies are available in the R package evd (fbvpot by Chris Ferro (2015)). From Falk and Guillou (2008), we know that peak over threshold method on random variable $\mathrm{U}$ provides polynomial generalized Pareto distribution for any $x_{0}$ with $1+\log \left(G\left(x_{0}\right)\right) \in$ $(0,1)$ i.e. $P\left(U>t x_{0} \mid U>x_{0}\right)=t^{-\alpha}, t \geq 1$ where $G(\cdot)$ is the distribution function of $U$. We choose appropriate $t$ and $x_{0}$ so that data should behave like near Pareto distribution. 


\begin{tabular}{|c|c|c|c|c|}
\hline $\begin{array}{c}\mathrm{n} \\
\text { parameters }\end{array}$ & 150 & 250 & 350 & 450 \\
\hline$\mu_{1}$ & {$[0.0001,0.01929]$} & {$[0.000077,0.01248]$} & {$[0.000067,0.00792]$} & {$[0.0000449,0.00619]$} \\
$\mu_{2}$ & {$[0.000034,0.004952]$} & {$[0.0000265,0.00337]$} & {$[0.0000203,0.002219]$} & {$[0.0000155,0.00175]$} \\
$\sigma_{1}$ & {$[0.5872,1.4421]$} & {$[0.6316,1.4810]$} & {$[0.6921,1.4760]$} & {$[0.71373,1.3677]$} \\
$\sigma_{2}$ & {$[0.2497,0.9518]$} & {$[0.2876,0.9674]$} & {$[0.3277,0.8631]$} & {$[0.33937,0.79859]$} \\
$\alpha_{0}$ & {$[0.6146,1.3454]$} & {$[0.6684,1.2655]$} & {$[0.7089,1.2288]$} & {$[0.75384,1.17617]$} \\
$\alpha_{1}$ & {$[0.1340,0.8017]$} & {$[0.1608,0.7578]$} & {$[0.1772,0.7227]$} & {$[0.19451,0.66522]$} \\
$\alpha_{2}$ & {$[0.7949,3.0734]$} & {$[0.9092,3.0089]$} & {$[0.9932,2.5686]$} & {$[1.03731,2.455004]$} \\
\hline
\end{tabular}

Table 7 Parametric Bootstrap confidence interval for $\mu_{1}=0, \mu_{2}=0, \sigma_{1}=1, \sigma_{2}=0.5, \alpha_{0}=1, \alpha_{1}=0.3$ and $\alpha_{2}=1.4$ in Modified Approach 2 (T)

\begin{tabular}{|c|c|c|c|c|}
\hline $\mathrm{n}$ & 150 & 250 & 350 & 450 \\
parameters & & & & \\
\hline$\mu_{1}$ & {$[0.000108,0.01929]$} & {$[0.000077,0.01248]$} & {$[0.000067,0.00791]$} & {$[0.000044,0.0061]$} \\
$\mu_{2}$ & {$[0.0000343,0.00495]$} & {$[0.0000265,0.00337]$} & {$[0.0000203,0.0022]$} & {$[0.000015,0.0017]$} \\
$\sigma_{1}$ & {$[0.5872,1.7322]$} & {$[0.6316,0.1 .6122]$} & {$[0.69207,1.5152]$} & {$[0.7137,1.3751]$} \\
$\sigma_{2}$ & {$[0.2496,1.1774]$} & {$[0.2877,1.0454]$} & {$[0.3277,0.8711]$} & {$[0.3394,0.7986]$} \\
$\alpha_{0}$ & {$[0.5967,1.3852]$} & {$[0.6684,1.2719]$} & {$[0.7089,1.2281]$} & {$[0.7538,1.1762]$} \\
$\alpha_{1}$ & {$[0.1346,0.9461]$} & {$[0.1585,0.8536]$} & {$[0.1773,0.7503]$} & {$[0.1945,0.6796]$} \\
$\alpha_{2}$ & {$[0.7951,3.7727]$} & {$[0.9093,3.2908]$} & {$[0.9932,2.5707]$} & {$[1.0373,2.4596]$} \\
\hline
\end{tabular}

Table 8 Parametric Bootstrap confidence interval for $\mu_{1}=0, \mu_{2}=0, \sigma_{1}=1, \sigma_{2}=0.5, \alpha_{0}=1, \alpha_{1}=0.3$ and $\alpha_{2}=1.4$ in Modified Approach 2 (WT)

\begin{tabular}{|c|c|c|c|c|}
\hline $\mathrm{n}$ & 150 & 250 & 350 & 450 \\
parameters & & & & \\
\hline$\mu_{1}$ & {$[0.000108,0.019]$} & {$[0.000077,0.0125]$} & {$[0.000067,0.0079]$} & {$[0.000045,0.0062]$} \\
$\mu_{2}$ & {$[0.000034,0.0049]$} & {$[0.000026,0.00337]$} & {$[0.0000203,0.0022]$} & {$[0.000016,0.0017]$} \\
$\sigma_{1}$ & {$[0.5846,1.1874]$} & {$[0.6355,1.6567]$} & {$[0.6956,1.5428]$} & {$[0.7161,1.3938]$} \\
$\sigma_{2}$ & {$[0.2406,1.2967]$} & {$[0.2875,1.0817]$} & {$[0.3277,0.8795]$} & {$[0.3393,0.8067]$} \\
$\alpha_{0}$ & {$[0.5921,1.4047]$} & {$[0.6656,1.2716]$} & {$[0.7075,1.2319]$} & {$[0.7520,1.1790]$} \\
$\alpha_{1}$ & {$[0.1378,0.9898]$} & {$[0.1621,0.8843]$} & {$[0.1798,0.7653]$} & {$[0.1946,0.6894]$} \\
$\alpha_{2}$ & {$[0.8007,4.127]$} & {$[0.9103,3.4069]$} & {$[0.9941,2.6199]$} & {$[1.0376,2.4805]$} \\
\hline
\end{tabular}

Table 9 Parametric Bootstrap confidence interval for $\mu_{1}=0, \mu_{2}=0, \sigma_{1}=1, \sigma_{2}=0.5, \alpha_{0}=1, \alpha_{1}=0.3$ and $\alpha_{2}=1.4$ in Modified Approach 3

\begin{tabular}{|c|c|c|c|c|}
\hline $\begin{array}{c}\mathrm{n} \\
\text { parameters }\end{array}$ & 150 & 250 & 350 & 450 \\
\hline$\mu_{1}$ & {$[0.0001,0.0193]$} & {$[0.000077,0.0125]$} & {$[0.000067,0.00791]$} & {$[0.000044,0.0062]$} \\
$\mu_{2}$ & {$[0.000034,0.0050]$} & {$[0.000026,0.00337]$} & {$[0.0000203,0.0022]$} & {$[0.000015,00017]$} \\
$\sigma_{1}$ & {$[0.5821,1.7914]$} & {$[0.6332,1.6449]$} & {$[0.6936,1.5209]$} & {$[0.7136,1.3763]$} \\
$\sigma_{2}$ & {$[0.2469,1.2961]$} & {$[0.2874,1.0815]$} & {$[0.3246,0.8798]$} & {$[0.3373,0.8037]$} \\
$\alpha_{0}$ & {$[0.6102,1.4071]$} & {$[0.6792,1.2729]$} & {$[0.7124,1.2345]$} & {$[0.7562,1.1791]$} \\
$\alpha_{1}$ & {$[0.1261,0.9899]$} & {$[0.1518,0.8474]$} & {$[0.1737,0.7367]$} & {$[0.1892,0.6740]$} \\
$\alpha_{2}$ & {$[0.7934,4.1231]$} & {$[0.9044,3.4036]$} & {$[0.9831,2.6059]$} & {$[1.0284,2.4709]$} \\
\hline
\end{tabular}

Table 10 Parametric Bootstrap confidence interval for $\mu_{1}=0, \mu_{2}=0, \sigma_{1}=1, \sigma_{2}=0.5, \alpha_{0}=1, \alpha_{1}=0.3$ and $\alpha_{2}=1.4$ in Modified Approach 4 
We assume the data to follow near equal to singular Marshall Olkin bivariate Pareto and try to verify our assumption. We fit the empirical survival functions with the marginals of this bivariate Pareto whose parameters can be obtained from the EM algorithm that we have developed. Figure-3 shows a good fit for both the marginals. The data analysis is performed based on sample size 1500 .

Empirical two dimensional density plot in Figure-4 verifies that Marshall-Olkin Pareto can be an alternative model for the transformed dataset. Parameter estimates of this bivariate distribution based on sample size $n=468$ are provided in Table11 and Table-12 whereas parametric bootstrap confidence intervals are provided in Table-13 and Table-14 respectively. All algorithms are used separately to calculate the estimates and confidence intervals.

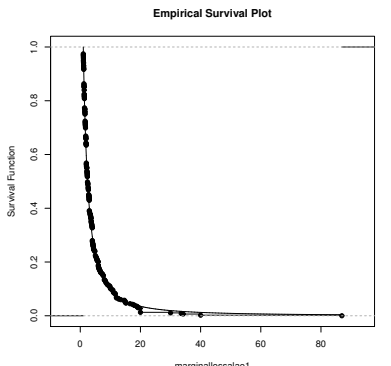

(a) $\xi_{1}$

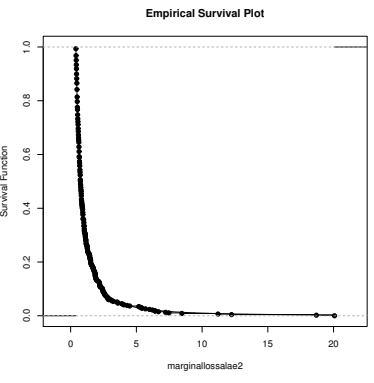

(b) $\xi_{2}$

Fig. 3 Survival plots for two marginals of the transformed dataset

Surface Plot of empirical density

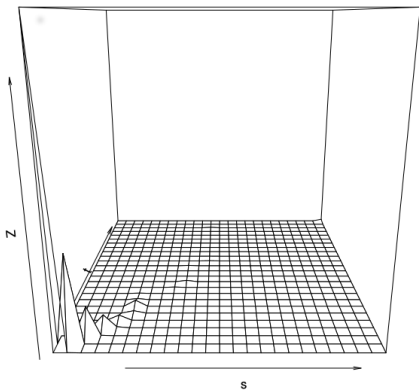

Fig. 4 Two dimensional density plots of the transformed dataset 


\begin{tabular}{|c|c|c|c|c|}
\hline Procedures & parameters & $\mu_{1}$ & $\mu_{2}$ & $\sigma_{1}$ \\
\hline Mod. 1 & & 1 & 0.4 & 2.5594 \\
Mod. 2 (T) & & 1 & 0.4 & 2.4936 \\
Mod. 2(WT) & & 1 & 0.4 & 2.9459 \\
Mod. 3 & & 1 & 0.4 & 3.2459 \\
Mod. 4 & & 1 & 0.4 & 3.1802 \\
\hline
\end{tabular}

Table 11 EM estimates of the parameters - Data Analysis

\begin{tabular}{|c|c|c|c|c|}
\hline Procedures & $\sigma_{2}$ & $\alpha_{0}$ & $\alpha_{1}$ & $\alpha_{2}$ \\
\hline Mod. 1 & 0.7176 & 0.7557 & 0.8162 & 0.9792 \\
Mod. 2 (T) & 0.8164 & 0.759 & 0.7878 & 1.1267 \\
Mod. 2 (WT) & 0.8162 & 0.7960 & 0.9209 & 1.0895 \\
Mod. 3 & 0.8163 & 0.8171 & 1.0100 & 1.0684 \\
Mod. 4 & 0.7947 & 0.8330 & 0.9667 & 1.0158 \\
\hline
\end{tabular}

Table 12 EM estimates of the parameters - Data Analysis

\begin{tabular}{|c|c|c|c|c|}
\hline & parameters & $\mu_{1}$ & $\mu_{2}$ & $\sigma_{1}$ \\
Procedures & & & & \\
\hline Mod. 1 & & {$[1.00013,1.0127]$} & {$[0.40002,0.4031]$} & {$[1.5793,2.9953]$} \\
Mod. 2(T) & & {$[1.00013,1.0127]$} & {$[0.40002,0.4031]$} & {$[1.4952,2.7412]$} \\
Mod. 2(WT) & & {$[1.0001,1.0135]$} & {$[0.40002,0.4032]$} & {$[1.5033,3.9036]$} \\
Mod. 3 & & {$[1.00013,1.0141]$} & {$[0.40002,0.4032]$} & {$[2.3578,4.9274]$} \\
Mod. 4 & & {$[1.00013,1.01397]$} & {$[0.40002,0.4032]$} & {$[2.3343,4.7869]$} \\
\hline
\end{tabular}

Table 13 Confidence interval - Data Analysis

\begin{tabular}{|c|c|c|c|c|}
\hline & $\sigma_{2}$ & $\alpha_{0}$ & $\alpha_{1}$ & $\alpha_{2}$ \\
Procedures & & & & \\
\hline Mod. 1 & {$[0.4554,0.9018]$} & {$[0.5435,0.8524]$} & {$[0.5378,1.0407]$} & {$[0.6726,1.3616]$} \\
Mod. 2 (T) & {$[0.4337,1.1601]$} & {$[0.5234,0.8712]$} & {$[0.4883,0.9623]$} & {$[0.6829,1.7491]$} \\
Mod. 2(WT) & {$[0.4448,1.1833]$} & {$[0.5445,0.9308]$} & {$[0.5090,1.3183]$} & {$[0.6909,1.7151]$} \\
Mod. 3 & {$[0.5731,1.2017]$} & {$[0.5869,0.9988]$} & {$[0.7788,1.6505]$} & {$[0.7996,1.7046]$} \\
Mod. 4 & {$[0.5658,1.1673]$} & {$[0.6089,1.0025]$} & {$[0.7526,1.5866]$} & {$[0.7770,1.6220]$} \\
\hline
\end{tabular}

Table 14 Confidence interval - Data Analysis

\section{Conclusion}

We use different variations of EM algorithms for estimating the parameters of singular bivariate Pareto distribution. All variations work quite well even for moderate sample size (say, 150, 250 etc). Our algorithms outperforms the current state-of-art algorithm by Asimit et al. The data analysis makes the paper more interesting. There was no discussion of any real-life data analysis in the previous paper. We have also calculated parametric bootstrap confidence interval which is absent in Asimit et al. The algorithm shown here can be applicable to other higher dimensional distributions with location and scale parameters. Given a real-life data set model selection can be 
an interesting issue among different other form of bivariate pareto distributions. More work is needed in this direction too.

A

A. 1

$$
\begin{gathered}
S\left(x_{1}, x_{2}\right)=P\left(X_{1} \geq x_{1}, X_{2} \geq x_{2}\right)= \begin{cases}S_{1}\left(x_{1}, x_{2}\right) & \text { if } \frac{x_{1}-\mu_{1}}{\sigma_{1}}<\frac{x_{2}-\mu_{2}}{\sigma_{2}} \\
S_{2}\left(x_{1}, x_{2}\right) & \text { if } \frac{x_{1}-\mu_{1}}{\sigma_{1}}>\frac{x_{2}-\mu_{2}}{\sigma_{2}} \\
S_{0}(x) & \text { if } \frac{x_{1}-\mu_{1}}{\sigma_{1}}=\frac{x_{2}-\mu_{2}}{\sigma_{2}}=x\end{cases} \\
S_{1}\left(x_{1}, x_{2}\right)=\left(1+\frac{\left(x_{1}-\mu_{1}\right)}{\sigma_{1}}\right)^{-\alpha_{1}}\left(1+\frac{x_{2}-\mu_{2}}{\sigma_{2}}\right)^{-\left(\alpha_{0}+\alpha_{2}\right)} \\
S_{2}\left(x_{1}, x_{2}\right)=\left(1+\frac{\left(x_{1}-\mu_{1}\right)}{\sigma_{1}}\right)^{-\left(\alpha_{0}+\alpha_{1}\right)}\left(1+\frac{x_{2}-\mu_{2}}{\sigma_{2}}\right)^{\alpha_{2}} \\
S_{0}(x)=(1+x)^{-\left(\alpha_{0}+\alpha_{1}+\alpha_{2}\right)}
\end{gathered}
$$

From the above expressions we can get the marginal of $X_{1}$ and $X_{2}$ taking $x_{1} \rightarrow \mu_{1}$ and $x_{2} \rightarrow \mu_{2}$,

$$
\begin{aligned}
& S_{X_{1}}\left(x_{1}\right)= \begin{cases}\left(1+\frac{\left(x_{1}-\mu_{1}\right)}{\sigma_{1}}\right)^{-\left(\alpha_{0}+\alpha_{1}\right)} & \text { if } x_{1}>\mu_{1} \\
1 & \text { otherwise }\end{cases} \\
& S_{X_{2}}\left(x_{2}\right)= \begin{cases}\left(1+\frac{x_{2}-\mu_{2}}{\sigma_{2}}\right)^{-\left(\alpha_{0}+\alpha_{2}\right)} & \text { if } x_{1}>\mu_{1} \\
1 & \text { otherwise }\end{cases}
\end{aligned}
$$

A. 2

This implies

$$
P\left(\min \left\{X_{1}, X_{2}\right\} \geq x\right)=P\left(X_{1} \geq x, X_{2} \geq x\right)
$$

Since,

$$
P\left(X_{1}>x, X_{2}>x\right)=P\left(U_{0}>x, U_{1}>x, U_{2}>x\right)
$$

Therefore,

$$
X_{k}=\min \left\{U_{0}, U_{k}\right\}
$$

$$
\begin{aligned}
P\left(X_{1}>x, X_{2}>x\right) & =P\left(U_{0}>x, U_{1}>x, U_{2}>x\right) \\
& =\left(1+\frac{x-\mu}{\sigma}\right)^{-\alpha_{0}}\left(1+\frac{x-\mu}{\sigma}\right)^{-\alpha_{1}}\left(1+\frac{x-\mu}{\sigma}\right)^{-\alpha_{2}} \\
& =\left(1+\frac{x-\mu}{\sigma}\right)^{-\left(\alpha_{0}+\alpha_{1}+\alpha_{2}\right)}
\end{aligned}
$$

\section{A.3}

It is easy to show that maximum likelihood estimate of location parameter is $\mu=\min \left\{X_{1}, X_{2}, \cdots, X_{n}\right\}$. Rest two equations can be obtained by plug-in the estimates of $\mu$ and taking derivative of Log-likelihood with respect to $\sigma$ and $\alpha$. Therefore the details of the proof is omitted. 


\section{References}

Asimit, A. V., E. Furman, and R. Vernic (2010). On a multivariate pareto distribution. Insurance: Mathematics and Economics 46(2), 308-316. 1, 2

Asimit, A. V., E. Furman, and R. Vernic (2016). Statistical inference for a new class of multivariate pareto distributions. Communications in Statistics-Simulation and Computation 45(2), 456-471. 1, 2

Asimit, A. V., R. Vernic, and R. Zitikis (2013). Evaluating risk measures and capital allocations based on multi-losses driven by a heavy-tailed background risk: The multivariate pareto-ii model. Risks 1(1), 14-33.

Balakrishnan, N., R. C. Gupta, D. Kundu, V. Leiva, and A. Sanhueza (2011). On some mixture models based on the birnbaum-saunders distribution and associated inference. Journal of Statistical Planning and Inference 141(7), 2175-2190.

Block, H. W. and A. Basu (1974). A continuous bivariate exponential extension. Journal of the American Statistical Association 69(348), 1031-1037.

Campbell, J. I. and S. Austin (2002). Effects of response time deadlines on adults strategy choices for simple addition. Memory \& Cognition 30(6), 988-994.

D, K. (2012). On sarhan-balakrishnan bivariate distribution. Journal of Statistics Applications \& Probability 1(3), 163.

Dey, A. K. and D. Kundu (2012). Discriminating between bivariate generalized exponential and bivariate weibull distributions. Chilean Journal of Statistics 3(1), 93-110. 2

Falk, M. and A. Guillou (2008). Peaks-over-threshold stability of multivariate generalized pareto distributions. Journal of Multivariate Analysis 99(4), 715-734. 14

fbvpot by Chris Ferro, A. S. F. (2015). evd: Functions for extreme value distributions. 14

Gupta, R. D. and D. Kundu (1999). Theory \& methods: Generalized exponential distributions. Australian \& New Zealand Journal of Statistics 41(2), 173-188.

Hanagal, D. D. (1996). A multivariate pareto distribution. Communications in Statistics-Theory and Methods 25(7), 1471-1488. 2

Khosravi, M., D. Kundu, and A. Jamalizadeh (2015). On bivariate and a mixture of bivariate birnbaumsaunders distributions. Statistical Methodology 23, 1-17.

Kundu, D. and A. K. Dey (2009). Estimating the parameters of the marshall-olkin bivariate weibull distribution by em algorithm. Computational Statistics \& Data Analysis 53(4), 956-965. 2

Kundu, D. and R. D. Gupta (2009). Bivariate generalized exponential distribution. Journal of Multivariate Analysis 100(4), 581-593. 2

Kundu, D. and R. D. Gupta (2010). A class of absolutely continuous bivariate distributions. Statistical Methodology 7(4), 464-477.

Kundu, D., A. Kumar, and A. K. Gupta (2015). Absolute continuous multivariate generalized exponential distribution. Sankhya B 77(2), 175-206.

Marshall, A. W. (1996). Copulas, marginals, and joint distributions. Lecture Notes-Monograph Series, 213-222.

Marshall, A. W. and I. Olkin (1967). A multivariate exponential distribution. Journal of the American Statistical Association 62(317), 30-44. 1, 2

McLachlan, G. and D. Peel (2004). Finite mixture models. John Wiley \& Sons.

Mirhosseini, S. M., M. Amini, D. Kundu, and A. Dolati (2015). On a new absolutely continuous bivariate generalized exponential distribution. Statistical Methods \& Applications 24(1), 61-83.

Nelsen, R. B. (2007). An introduction to copulas. Springer Science \& Business Media. 2

Rakonczai, P. and A. Zempléni (2012). Bivariate generalized pareto distribution in practice: models and estimation. Environmetrics 23(3), 219-227.

Ristić, M. M. and D. Kundu (2015). Marshall-olkin generalized exponential distribution. Metron 73(3), 317-333.

Sarhan, A. M. and N. Balakrishnan (2007). A new class of bivariate distributions and its mixture. Journal of Multivariate Analysis 98(7), 1508-1527. 2

Smith, R. L. (1994). Multivariate threshold methods. In Extreme Value Theory and Applications, pp. 225-248. Springer.

Smith, R. L., J. A. Tawn, and S. G. Coles (1997). evd : Functions for extreme value distributions. https://cran.r-project.org 84(2), 249-268.

Tsay, R. S. (2014). An introduction to analysis of financial data with R. John Wiley \& Sons. 
Yeh, H. C. (2000). Two multivariate pareto distributions and their related inferences. Bulletin of the Institute of Mathematics, Academia Sinica. 28(2), 71-86. 2

Yeh, H.-C. (2004). Some properties and characterizations for generalized multivariate pareto distributions. Journal of Multivariate Analysis 88(1), 47-60. 2 